\title{
Journal of the Saudi Heart Association
}

\section{Perioperative management of Wilms' tumor with intracardiac extension: Report of two cases with review of literature}

Follow this and additional works at: https://www.j-saudi-heart.com/jsha

Part of the Cardiology Commons

(c) (i) (9)

This work is licensed under a Creative Commons Attribution-Noncommercial-No Derivative Works 4.0 License.

\section{Recommended Citation}

Altwaeel, Hayan; Kabbani, Mohamed S.; Al Shammari, Ahmad; Al- Namshan, Mohammed; and Alghamdi, Abdullah A. (2020) "Perioperative management of Wilms' tumor with intracardiac extension: Report of two cases with review of literature," Journal of the Saudi Heart Association: Vol. 32 : Iss. 1 , Article 19. Available at: https://doi.org/10.37616/2212-5043.1018

This Case Report is brought to you for free and open access by Journal of the Saudi Heart Association. It has been accepted for inclusion in Journal of the Saudi Heart Association by an authorized editor of Journal of the Saudi Heart Association. 


\title{
Perioperative management of Wilms' tumor with intracardiac extension: Report of two cases with review of literature
}

\author{
Hayan Altwaeel ${ }^{a}$, Mohamed S. Kabbani ${ }^{\text {a,e,*, Ahmad Al Shammari }}{ }^{\text {c,e }}$, \\ Mohammed Al-Namshan ${ }^{\mathrm{d}, \mathrm{e}}$, Abdullah A. Alghamdi ${ }^{\mathrm{b}, \mathrm{e}}$

\footnotetext{
a Section of Pediatric Cardiac Intensive Care, Department of Cardiac Sciences, King Abdulaziz Medical City, Riyadh, Saudi Arabia

${ }^{\mathrm{b}}$ Section of Cardiac Surgery, Department of Cardiac Sciences, King Abdulaziz Medical City, Riyadh, Saudi Arabia

' Section of Urology Surgery, Department of Surgery, King Abdulaziz Medical City. Riyadh, Saudi Arabia

${ }^{\mathrm{d}}$ Department of Pediatric Surgery, King Abdullah Specialist Children Hospital, Riyadh, Saudi Arabia
} \\ ${ }^{\mathrm{e}}$ King Saud Bin AbdulAziz University for Health Sciences, Riyadh, Saudi Arabia
}

\begin{abstract}
Wilms' tumor (WT) is one of the solid tumor that affects children. It involves the kidney and may extend to the lungs and liver. WT conquers the adjacent blood vessels such as renal veins and inferior vena cava (IVC); its extension to IVC and right-sided heart chambers is unusual. Furthermore, when the tumor extends to heart chambers, its surgical management becomes challenging and demands multidisciplinary medical and surgical specialties including pediatric cardiac surgery. In this report, we discuss the surgical management and perioperative treatment of two unusual cases of WT with IVC and intracardiac extension.
\end{abstract}

Keywords: Intracardiac extension, Surgical management, Wilms' tumor

\section{Introduction}

W ilms' tumor (WT) or nephroblastoma is the most common genitourinary malignancy in children [1]. The overall outcome of WT is generally good. Recently, a significant success has been achieved by increasing the overall 5-year survival rates, with the expectation that the majority of patients with WT will be cured following multimodal treatment that includes surgical resection with total nephrectomy, chemotherapy, and radiotherapy. The overall prognosis of WT has improved significantly and the 5 -year survival rate is now over $90 \%$ in recent years $[2,3]$.

Intravascular expansion of WT in a shape of tumor thrombus is unusual. Due to its high tendency to conquer the blood vessels, the invasion of tumor thrombus of WT to the inferior vena cava (IVC) is a well-recognized phenomenon [4]. While the incidence of expansion into the IVC is reported in only $4-8 \%$ of patients, intra-atrial thrombus of WT occurs in approximately $1-3 \%$ of patients $[5,6]$. Furthermore, the expansion of WT through the tricuspid valve (TV) into the right ventricle (RV) is a rare occurrence [5].

The incidence of WT thrombus was reported to be higher in the right side than in the left side due to shorter right renal vein [2]. Management of WT requires removing all components of the tumor including intracardiac extension. This requires a multidisciplinary approach in the management with the adjunct use of cardiopulmonary bypass to ensure the removal of all intracardiac components of the tumor, particularly when there is RV involvement.

Received 27 June 2019; revised 21 October 2019; accepted 23 October 2019.

Available online 17 April 2020

* Corresponding author at: Pediatric Cardiac ICU, King AbdulAziz Medical City, Cardiac Science Department, 1423. P. O. Box 22490, Riyadh 11426, Saudi Arabia.

E-mail address: kabbanim@ngha.med.sa (M.S. Kabbani). 
In this report, we present two unusual pediatric cases who had WT with intracardiac extension. The tumor was surgically removed in both, including excision of all intracardiac metastases while the patients were supported with cardiopulmonary bypass. The report will discuss perioperative cardiac management following removal of the main tumor and its cardiac extension.

\section{Cases reports}

\subsection{Case 1}

A 32-month-old female receiving neoadjuvant chemotherapy for stage IV left-sided WT with intracardiac WT thrombus extension associated with minimal tumor response to therapy.

Her abdomen and chest computed tomography (CT) scan revealed a large left renal mass of WT with tumor thrombus extending throughout the left renal vein and IVC into the right atrium, along with abdominal lymphadenopathy and hepatomegaly with no clear evidence of liver metastasis (Fig. 1).

She underwent combined sternotomy and upper laparotomy for left radical nephrectomy. Extraction of IVC and intracardiac thrombus was performed by general pediatric and pediatric cardiac surgical teams.

The procedure was started by a transverse upper abdominal laparotomy for left-sided radical nephrectomy. Then, the cardiac surgery team performed a midline sternotomy. The cardiopulmonary bypass was instituted, aortic cross-clamp was applied, and antegrade cardioplegia was administered; subsequently, the tumor was retrieved and excised in one piece through the right atrium.

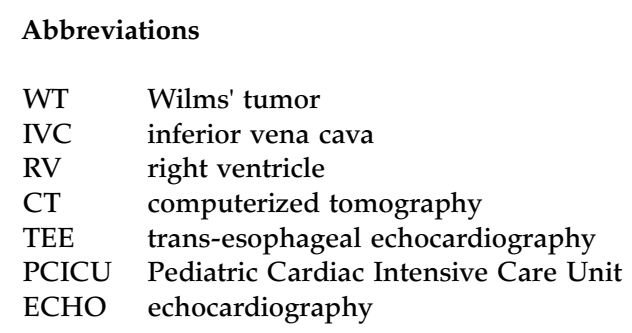

During surgery, the IVC was scanned by transesophageal echocardiography (TEE) that neither showed residual lesions in the heart nor near atrialIVC junction.

Postoperatively, she recovered smoothly in the Pediatric Cardiac Intensive Care Unit (PCICU) with no significant bleeding. Her postoperative management included prophylactic cefazolin and anticoagulation. Once chest tube drainage cleared from bloody to hemoserous fluid, enoxaparin was initiated to prevent venous thrombosis related to manipulation and total extraction of IVC thrombus. Enoxaparin prophylaxis was continued until hospital discharge. Patient was noted to develop hypertension in the perioperative period that required sodium nitroprusside infusion in the immediate postoperative stage and amlodipine upon home discharge. Chemotherapy was resumed 2 weeks after surgery; on the third postoperative week, adjunct radiotherapy was commenced. Post resection echo demonstrated complete resection of intracardiac tumor. We observed no significant arrhythmia during the postoperative period, and cardiac function was preserved.
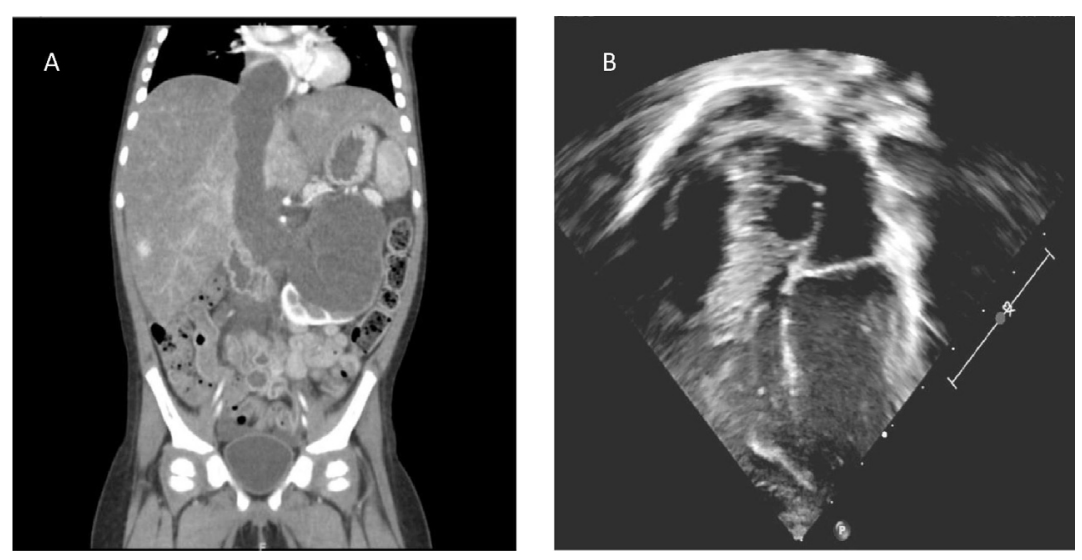

Fig. 1. Left-sided Wilms' tumor extending through the inferior vena cava and filling the right atrium: appearance on computed tomography imaging (A) and echocardiography (B). 


\subsection{Case 2}

A 13-month-old boy diagnosed to have stage IV right-sided WT with extensive IVC thrombosis and right atrial WT thrombus. After initiation of neoadjuvant chemotherapy, removal of intracardiac thrombus was undertaken.

Abdominal CT scan showed a large right renal mass of WT with tumor thrombus extending throughout the right renal vein and IVC into the right atrium. Furthermore, echocardiography showed tumor extension to the RV through TV (Fig. 2).

He underwent right-sided radical nephrectomy, excision of intracaval tumor thrombus, and IVC reconstruction through the joint procedure of pediatric cardiac surgery and pediatric urology.

The surgery was started with median sternotomy performed by pediatric cardiac surgeon. Then, the pediatric urology surgeon extended the incision to median laparotomy. Next, tumor dissection and radical nephrectomy were performed, and the patient was subsequently connected to cardiopulmonary bypass. Aortic crossclamp was applied, and antegrade cardioplegia was administered. The right atrium was opened and large ventricular, atrial, and IVC tumor was retrieved and excised in one piece. Intraoperatively, TEE showed no residual lesions.

After surgery, the patient had a smooth course in PCICU. Sodium nitroprusside was started during surgery and continued postoperatively for antihypertensive management. To prevent IVC thrombosis, enoxaparin was initiated 24 hours after surgery and continued until hospital discharge. He was discharged 3 days after surgery to the cardiac ward in stable hemodynamic and respiratory condition, and follow-up echo studies post-surgery showed moderate TV regurgitation with good reserved cardiac function. Chemotherapy and radiotherapy were scheduled 3 weeks after surgery.

\section{Discussion}

The presence of intracardiac extension of WT requires special surgical preparation for removal that increases the complexity and morbidity of surgical resection.

Removal of cardiac extension requires exploration of right heart cavities. Complete arrest of the heart while the patient is supported by cardiopulmonary bypass will facilitate complete resection.

Postoperative cardiac complications such as dysrhythmia, venous thromboembolisms, bleeding, or infection may complicate this major surgery and require immediate recognition and management $[7,8]$.

The management plan for these cases requires a multidisciplinary approach that may include pediatric cardiology, cardiac surgeon, general pediatric surgeon, urologist, and pediatric oncology. Furthermore, using TEE preoperatively and intraoperatively is very helpful to evaluate vascular tumor extension and to ensure complete resection during surgery with no residual intracardiac thrombus $[7,8]$.

Both of our patients showed no specific cardiac symptoms before surgery, indicating the importance of preoperative ultrasound, CT scan, and echocardiography screening for tumor grading, evaluation of intravascular tumor extension, and facilitation of proper multidisciplinary surgical planning.
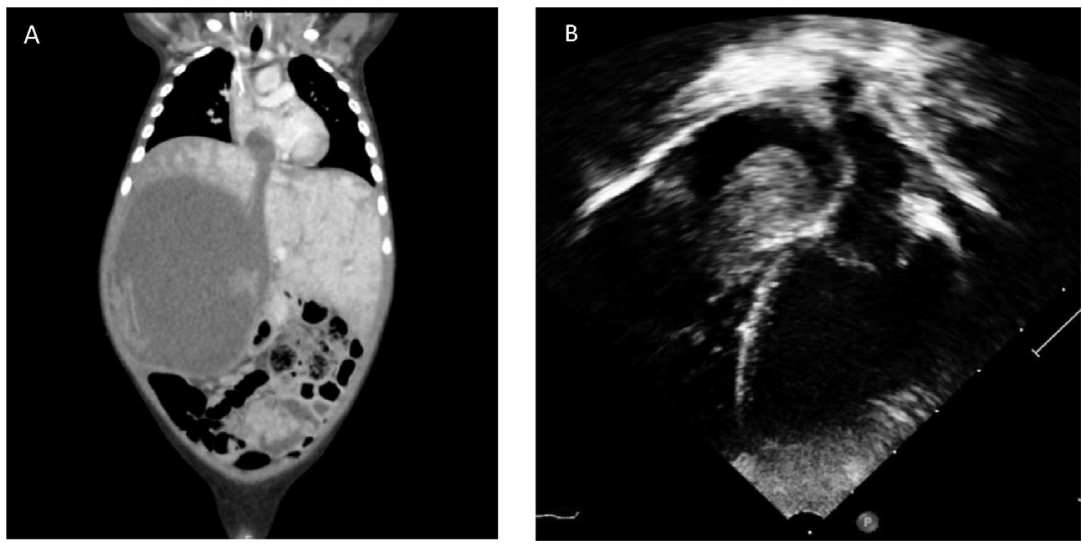

Fig. 2. Right-sided Wilms' tumor extending and filling the inferior vena cava and the right atrium with extension through the tricuspid valve as demonstrated by computed tomography imaging (A) and echocardiography (B). 
Preoperative chemotherapy is recommended for regression of intracardiac tumor thrombus [6]. Both of our patients received chemotherapy preoperatively but continued to show residual tumor thrombus. Patient 1 had persistent tumor in the right atrium and Patient 2 had persistent tumor in the right atrium and RV. Both of them had no intraoperative complications, and their postoperative course was smooth apart from severe systemic hypertension and sinus tachycardia. Both patients were free of the expected arrhythmia typically seen in cardiac surgery involving the right atrium.

Patients received cefazolin as prophylaxis for 48 hours after surgery and were transferred out of PCICU to continue their scheduled chemotherapy and radiotherapy $2-3$ weeks after surgery.

\section{Conclusion}

Intracardiac extension of $\mathrm{WT}$ is rare but warrants screening for all cases by combination of imaging including echocardiography, ultrasonography, and CT scan. Presence of WT thrombus inside the right heart chambers require exploration of the heart with the assistance of a cardiac surgeon in a center equipped with cardiopulmonary bypass to remove the extension and ensure maximal or entire resection of the tumor. The prognosis of these cases depends on primary pathology, but cardiac recovery is good provided complete resection of the tumor and its intracardiac extension is achieved.

\section{Conflicts of interest}

The authors declare no conflicts of interest.

\section{References}

[1] Dénes FT, Duarte RJ, Cristófani LM, Lopes RI. Pediatric genitourinary oncology. Front Pediatr 2013;1:48.

[2] Dome JS, Fernandez CV, Mullen EA, Kalapurakal JA, Geller JI, Huff V, et al. Children's Oncology Group's 2013 Blueprint for research: renal tumors. Pediatr Blood Cancer 2013;60:994-1000.

[3] Chintagumpala M, Muscal JA. Presentation, diagnosis, and staging of Wilms tumor. In: Pappo ES, editor. Upto Date. Wolters Kluwer; 2013.

[4] Emir S. Wilms tumor with intravascular tumor thrombus. Transl Pediatr 2014;3:29-33.

[5] Lall A, Pritchard-Jones K, Walker J, Hutton C, Stevens S, Azmy A, et al. Wilms' tumor with intracaval thrombus in the UK Children's Cancer Study Group UKW3 trial. J Pediatr Surg 2006;41:382-7.

[6] Abdullah Y, Karpelowsky J, Davidson A, Thomas J, Brooks A, Hewitson J, et al. Management of nine cases of Wilms' tumor with intra-cardiac extension-a single center experience. J Pediatr Surg 2003;48:394-9.

[7] Khozeimeh N, Sinha P, Dome JS, Guzzetta Jr PC. Strategy for management of retroperitoneal tumors with caval tumor thrombus. J Pediatr Surg 2011;46:2065-70.

[8] Cox SG, Davidson A, Thomas J, Brooks A, Hewitson J, Numanoglu A, et al. Surgical management and outcomes of 12 cases of Wilms tumor with intracardiac extension from a single center. Pediatr Surg Int 2018;34:227-35. 